\title{
Modeling of the high-strength clinker-free binder structure
}

\author{
Victoria Petropavlovskaya ${ }^{1, *}$, Tatiana Novichenkova ${ }^{1}$, Kirill Petropavlovskii ${ }^{2}$, and \\ Aleksandr Buryanov ${ }^{2}$ \\ ${ }^{1}$ TvSTU, Department of building products and constructions, 170026, Af. Nikitin 22, Russia \\ ${ }^{2}$ MGSU, Department of binders and concretes, Yaroslavskoe shosse, 26, Russia
}

\begin{abstract}
Modern approaches in materials science should provide a solution to the problems of energy and resource conservation. They are also called upon to develop a line of directed synthesis of composites that possess the required parameters over the entire spectrum of properties. In order to obtain a high-strength structure of a clinker-free binder, the possibility of obtaining it on the basis of natural gypsum stone is considered. As is known, the strength of crystallization structures by adhesion in contact and the number of contacts is determined. To optimize the internal structure of a high-strength clinker-free binder, to obtain the maximum number of contacts and increase their strength, the possibilities of geometric topological approaches were used. When solving such problems, the geometric parameters and organization levels of the structures of disperse systems allow topology to be described. The fundamental possibility of obtaining a high-strength composite structure through the prism of the formation of a dispersed system with a maximum number of phase contacts between crystals of calcium sulfate dihydrate is considered. The granulometric composition of gypsum stone must meet the requirements (principles) of hardening, physical-chemical model and type of structure. Therefore, in the work, the possibility of obtaining high binder strength by optimizing it in accordance with the model was studied. mixtures of two powders with different average particle sizes were used. This increases efficiency and improves the properties of the binder.
\end{abstract}

\section{Introduction}

Modern approaches in materials science should provide a solution to the problems of energy and resource conservation. They also develop a line of directed synthesis of new type composites. Such composites possess the required parameters over the entire spectrum of properties [1, 2].

In order to synthesize the structural strength of a clinker-free binder based on gypsum stone, condensation hardening requires the formation of special crystallization conditions. Certain conditions are required. They should fundamentally provide the possibility of synthesis of such a structure. High-strength clinker-free binder have such a crystallization

\footnotetext{
*Corresponding author: victoriapetrop@gmail.com
} 
structure. Hardening is possible in it due to the development of internal stresses. The process of structure formation can be controlled only if the optimal particle size distribution of the dispersed clinker-free system is maintained. Optimization of the internal structure of the high-strength clinker-free binder will strengthen contacts.

As you know, in physical chemistry distinguish between coagulation and crystallization types of spatial structures [3]. They are formed by particles of matter under the influence of various conditions [4]. If the former are the result of exposure, for example, by simple contact, the latter develops during isothermal distillation, pressing or sintering, as well as during the synthesis of a new finely divided solid phase from supersaturated solutions or melts. Such contact crystallization structures can be implemented, for example, in polymeric or mineral materials, binders [3]. Coagulation contacts formed in pastes, in powders, are mechanically reversible, can decompose, for example, under the influence of external conditions. Their strength is low, ranging from $10^{-8}-10^{-7} \mathrm{~N}$ [3]. Crystallization or phase contacts, on the contrary, are irreversible, due to their significant contact area, have a high strength of $10^{-7}-10^{-6} \mathrm{H}$. The development of a contact from primary condensation to the maximum possible crystallization, ideally to complete crystal intergrowth, provides the structure with mechanical strength [5, 6].

When considering crystallization structures, the strength is determined by the adhesion in the contact and the number of contacts, as is known [3].

Each structure is characterized by a certain dispersion and, therefore, the number of contacts per $1 \mathrm{~cm} 2$ of area [4]. It was established [3] that the number of contacts for disperse systems will be $-10^{2}-10^{3}$. If they consist of particles with sizes $\approx 0.1 \mathrm{~mm}$.

The number of contacts is closely related to the properties of disperse systems and depends on the shape, size of the particles, and the way they are packaged. The smaller the particle size and denser their packaging, the greater the strength of the structure. This is explained by an increase in the number of contacts per unit volume [3, 4, 7].

To optimize the internal structure of a high-strength clinker-free binder, it is necessary to use the capabilities of geometric (topological) approaches [4].

From the position of topology, well-known structures can be considered as objects of the implementation of self-organization mechanisms of systems of forming crystals [5, 8]. Elements of the system can crystallize into cubic or hexagonal structures. In the Periodic system, it is possible to distinguish areas with topologically different types of structures. G.D. Ilyushin [5] in them only two groups of elements are distinguished. Which crystallize in a cubic and hexagonal structure. Calcium, as an element of the periodic system, crystallizes in a cubic I-lattice. But after the first phase transition, it possesses a hexagonal lattice [5].

In order to study the possibility of synthesizing a clinker-free binder from gypsum stone of normalized composition, in this work the particle size distribution of the dispersed system and the properties of the structure of gypsum stone after molding and hardening were studied. mixtures of two powders with different average particle sizes were used (Fig. 1, 2). This efficiency improves and improves the properties of the binder. The dispersion system (clinker-free binder) is defined by the packing of particles as topological elements, with their ordered placement in hexagonal packaging.

\section{Materials and methods}

In the work, as a raw material component, a mixture of two gypsum powders was used. They were milled from natural gypsum stone in a ball mill (field - Shedoksk). The chemical composition of gypsum powders by gypsum stone is presented. The CaSO4 $42 \mathrm{H} 2 \mathrm{O}$ content is from 90.60 to $99.73 \%$. Separate gypsum stone layers are characterized by lower $\mathrm{CaSO}_{4} \cdot 2 \mathrm{H}_{2} \mathrm{O}$ contents - from 65.23 to $88.3 \%$, they have hydrated anhydrite [9]. 
The average chemical composition in table 1 is presented. Reflections correspond to an interplanar distance of 7.5943; 4.2756; 3.7937; 3.0616. This is the results of x-ray analysis show. Gypsum reflections are consistent.

Table 1. The chemical composition of gypsum stone.

\begin{tabular}{|c|c|}
\hline Component & Content, $\%$ \\
\hline $\mathrm{H}_{2} \mathrm{O}$ & 21,2 \\
\hline Insoluble residue & 18,5 \\
\hline $\mathrm{SO}_{3}$ & 50,4 \\
\hline $\mathrm{CaSO}_{4} \cdot 2 \mathrm{H}_{2} \mathrm{O}$ & 99,7 \\
\hline
\end{tabular}

The particle size distribution of the raw powders using a Fritsch Particle Sizer Analysette 22 type laser microanalyzer was evaluated. The determination of the particle size distribution of the studied samples by laser diffraction was carried out. According to the requirements of the standard ISO 13320-1: 2009 "Analysis of particle size. Laser diffraction methods" powders were evaluated. Coarse powder is characterized by an average particle size of $d 50=18.977$ microns, a maximum particle size of d99 $=130.957$ microns; a particle powder of less than 2 microns contains $10.07 \%$ by weight. An analysis of the composition of the powders was carried out. The differential and integral particle size distribution in the composition of the coarse powder is shown in Figure 1. A powder of a higher degree of grinding with an average particle size is characterized by $\mathrm{d} 50=4.733 \mu \mathrm{m}$, with a maximum particle size of $\mathrm{d} 99=38.030 \mu \mathrm{m}$; particles less than 2 microns in the composition of the powder contains $26.18 \%$ by weight. The particle size distribution is shown in Figure 2. The strength of the cylinder samples was also evaluated according to GOST requirements. They were obtained by the method of semi-dry pressing in a laboratory hydraulic press, and they were kept for 14 days. Raw mixtures of gypsum powder were manually mixed.

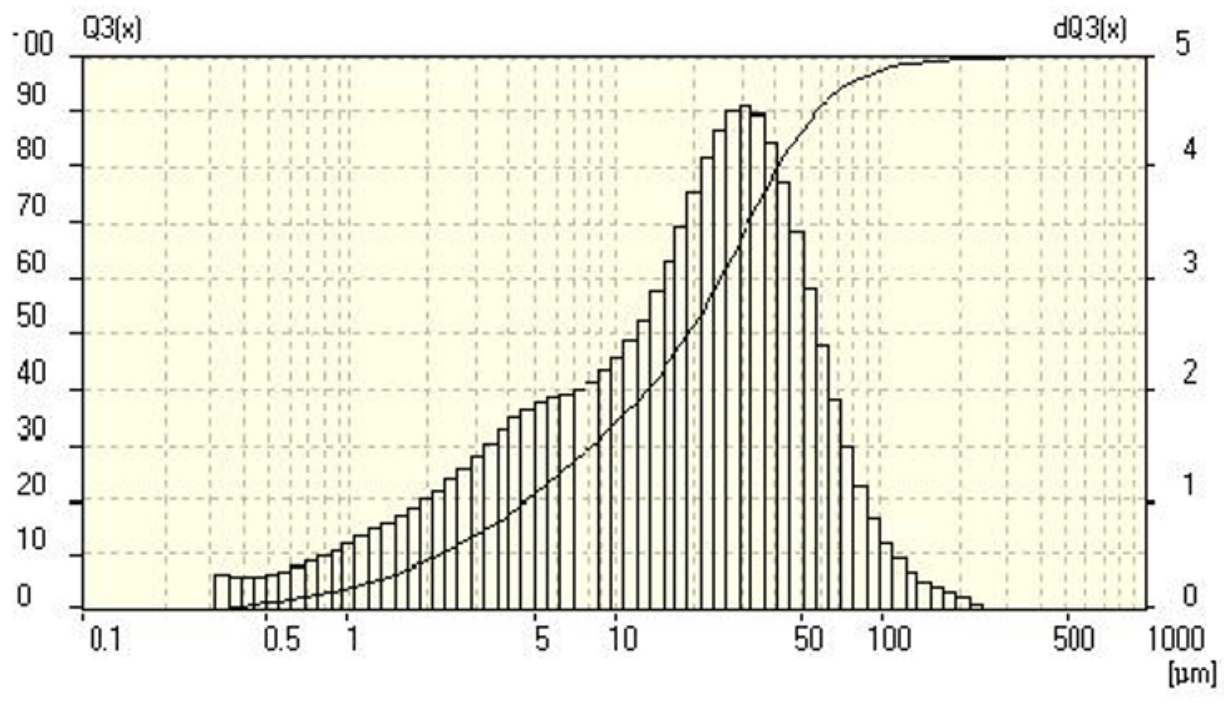

Fig. 1. Particle Size Distribution of Coarse Powde. 


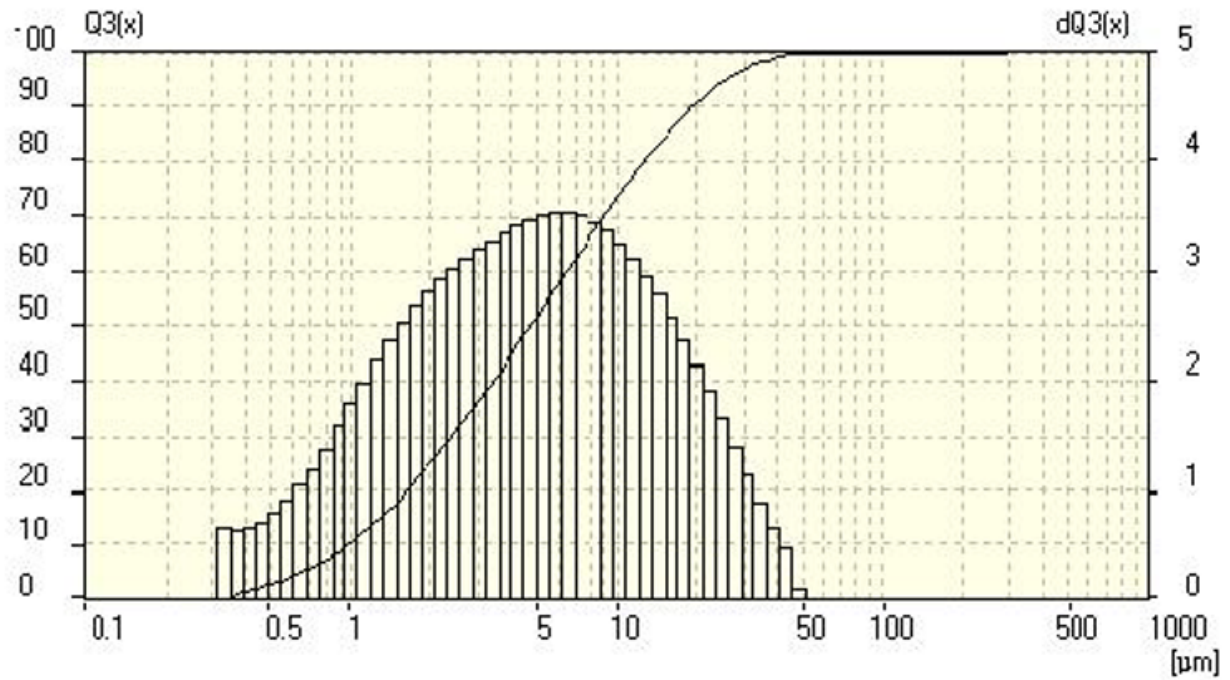

Fig. 2. Particle Size Distribution of Fine Powde.

\section{Results and discussion}

The results of the studies are shown below (Fig. 3). They show the effectiveness of regulating the composition of the mixtures. The introduction into the composition of the binary raw mix of two-water gypsum of fine grinding powder allows you to adjust the properties of the composite. The strength of the resulting composite to a large extent affects the water content in the feed mixture (W/S).

On the other hand, an increase in the number of fine particles in the mixture leads to an increase in the number of contacts between particles in a unit volume. This increases the strength of the composite structure (Fig. 3).

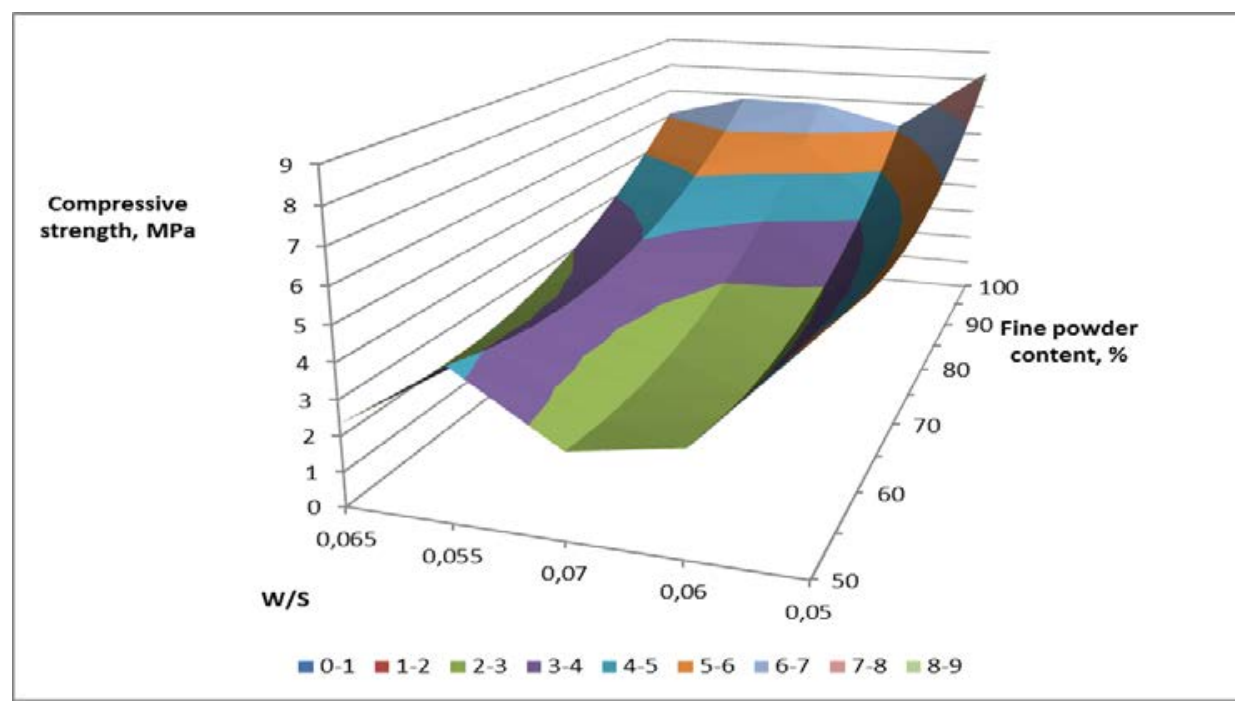

Fig. 3. The dependence of the strength of gypsum on the particle size distribution of mixtures and water content. 
By varying two factors (particle size distribution and water-solid ratio), the optimum result in the strength of the pressed composite can be obtained. In this case, the optimal result is determined by the strength value $(8.3 \mathrm{MPa})$. At a content of the second powder (fine grinding) in an amount of $50 \%$, it is observed in the composition of the raw mix of the non-calcined composite. The maximum strength of the non-fired composite at the age of 14 days - 8.2 MPa at a water content of $5 \%$ is achieved. However, the material may have an additional increase in strength by 28 days of hardening.

Thus, the standardization of the granulometric composition of the raw material mixture allows you to adjust the strength of the binder free binder. The physical and mechanical characteristics of the composite are used in the manufacture of building products for industrial and residential construction.

As well as for objects of household and social purpose, to which increased requirements for fire and environmental safety are imposed. Such gypsum-based binders will provide the construction industry with high-quality energy-efficient materials.

\section{References}

1. J. Sykut, Discrete element method (DEM) as a tool for investigating properties of granular materials, Pol. J. Food Nutr. Sci, 2, pp.179-173 (2007)

2. E.G. Shchukin, N.V. Arkhincheeva, Study of the properties of gypsum waste and the production of gypsum materials on their basis, Bulletin of the ESSUTM, 3, pp. 48 53 (2013).

3. E. Schukin, A.Pertsov, E. Amelina, Colloid chemistry, (Higher school, 2007)

4. V. Petropavlovskaya, T. Novichenkova, A. Buryanov, Hyperpressed Calcium Sulfate Dihydrate Structure, Advanced Materials Research, Vols. 1025 -1026, pp. 820-823. (2014)

5. G.D. Ilyushin, Modeling of self-organization processes in crystal-forming system, (URSS editorial, 2003)

6. V. Petropavlovskaya, A. Buryanov, A. Pustovgar, T. Novichenkova, K. Petropavlovskii, Mechanism of gypsum hardening, MATEC Web of Conferences, (2018)

7. V.I. Osipov, Clay tires of oil and gas fields, (Science, 2001)

8. N.F. Kosenko, N.V. Filatova, The effect of mechanical activation on the properties of pressed gypsum materials, University News. Chemistry and chemical technology, 9, pp. 99-100 (2007)

9. L.V. Dubovchenko, Mineralogical and technological features and prospects for industrial use of the Upper Jurassic sulfate rocks of titanium in the Western Ciscaucasia, Problems of geology and subsoil development, pp.187 - 189 (2015)

10. A. Satoh, Introduction to Practice of Molecular Simulation: Molecular Dynamics, Monte Carlo, Brownian Dynamics, Lattice Boltzmann and Dissipative Particle Dynamics, Elsevier, (2011) 